\title{
Spatiotemporal Rheochaos in Nematic Hydrodynamics
}

\author{
Buddhapriya Chakrabarti, ${ }^{1,2, *}$ Moumita Das, ${ }^{1, \dagger}$ Chandan Dasgupta, ${ }^{1,2}$ Sriram Ramaswamy, ${ }^{1,2}$ and A. K. Sood ${ }^{1, \sharp}$ \\ ${ }^{1}$ Department of Physics, Indian Institute of Science, Bangalore 560012, India \\ ${ }^{2}$ Condensed Matter Theory Unit, JNCASR, Bangalore 560064, India
}

\begin{abstract}
Motivated by recent observations of rheochaos in sheared wormlike micelles, we study the coupled nonlinear partial differential equations for the hydrodynamic velocity and order-parameter fields in a sheared nematogenic fluid. In a suitable parameter range, we find irregular, dynamic shear banding and establish by decisive numerical tests that the chaos we observe in the model is spatiotemporal in nature.
\end{abstract}

Wormlike micellar solutions of cetyltrimethylammonium tosylate (CTAT) show rheochaos [1-3]: for a suitable range of concentrations and temperatures, the stress vs shear-rate curve in $[1,3]$ shows a plateau signaling a rheological phase coexistence and, in the plateau region, the time series of the shear and normal stresses at constant shear rate show deterministic chaos. This is remarkable because it means that the rheological equation of state [4], that is, the relation between stress and shear rate, even for macroscopic experimental samples, has huge fluctuations about the mean [5]. A realistic description of the complex dynamics of micellar systems as well as of other rheological oscillations [6,7] may require variables specific to the system in question [8,9]. The studies of [10-12], however, are significant in that they find temporal rheochaos in the dynamics of the passively advected alignment tensor alone. They use the well-established equations of hydrodynamics for a nematic order parameter, outside the regime of stable flow alignment, but consider only spatially homogeneous states [13] and, hence, cannot explore the relation of the observed chaos to shear banding [14].

In this Letter we study numerically the equations of the traceless symmetric order parameter for a sheared nematogenic system, allowing for spatial variation. In the parameter range termed "complex" in the phase diagram of the studies $[11,12]$ of the spatially homogeneous situation, we find spatiotemporal chaos. We give evidence for this in Fig. 2 for the dynamic instability of shear bands, Fig. 5 for the spatial distribution of "stress drops," Fig. 1(b) for the local phase portrait in the chaotic regime, and, decisively, Fig. 6 for the number of positive Lyapunov exponents as a function of (sub)system size. We now present our model in detail and show how these results arise.

Models of complex rheology [4] generally postulate an equation of motion for the stress in addition to that for the velocity field. Our work was motivated in part by the failure [15] to find chaos in the the popular JohnsonSegalman (JS) [16] model for the coupled dynamics of the stress tensor and the hydrodynamic velocity field.
Since this model is linear in the stress, and contains bilinear products of velocity gradient and stress, it is natural to ask if nonlinearities in the stress are the missing ingredient. The JS equation is most naturally thought of as arising from the underlying dynamics of a local alignment tensor, the traceless, symmetric nematic order parameter $\mathbf{Q}$, measuring in this case the alignment of the micellar worms. The stress $\boldsymbol{\sigma}^{O P}$ [17] due to $\mathbf{Q}$ is defined via a free-energy functional $F[\mathbf{Q}][12,18-20]$. Nonlinearities arise naturally in nematodynamics from terms of order higher than $\mathbf{Q}^{2}$ in $F$. It is these that lead to the rich dynamical phase diagram, including a variety of routes to chaos, in the work of [10-12].

The alignment tensor obeys the equation of motion

$$
\begin{aligned}
\frac{\partial \mathbf{Q}}{\partial t}+\mathbf{u} \cdot \nabla \mathbf{Q}= & \tau^{-1} \mathbf{G}+\left(\alpha_{0} \mathbf{\kappa}+\alpha_{1} \mathbf{\kappa} \cdot \mathbf{Q}\right)_{S T} \\
& +\boldsymbol{\Omega} \cdot \mathbf{Q}-\mathbf{Q} \cdot \mathbf{\Omega},
\end{aligned}
$$

where $\mathbf{I}$ is the unit tensor, $\mathbf{u}$ is the hydrodynamic velocity field, $\quad \boldsymbol{\kappa} \equiv(1 / 2)\left[\nabla \mathbf{u}+(\boldsymbol{\nabla u})^{T}\right]$ and $\boldsymbol{\Omega} \equiv(1 / 2)[\nabla \mathbf{u}-$ $\left.(\nabla \mathbf{u})^{T}\right]$ are the shear-rate and vorticity tensors, respectively, $\tau$ is a bare relaxation time, $\alpha_{0}$ and $\alpha_{1}$ are parameters related to flow alignment, the subscript $S T$ denotes

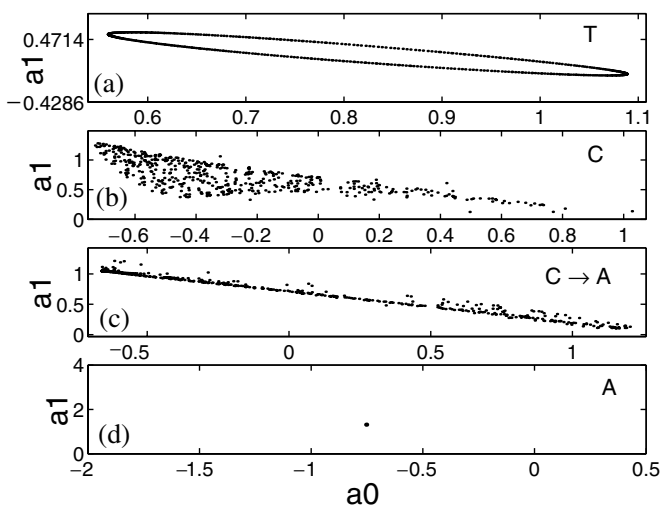

FIG. 1. Plots showing $a_{1}\left(x_{0}, t\right)$ vs $a_{0}\left(x_{0}, t\right)$ for tumbling (panel $\mathrm{T})$, chaotic $(\mathrm{C})$, onset of aligning $(\mathrm{C} \rightarrow \mathrm{A})$, and aligning (A) regimes. 
symmetrization and trace removal, and

$$
\begin{aligned}
\mathbf{G} \equiv- & (\delta F / \delta \mathbf{Q})_{S T} \\
=- & {\left[A \mathbf{Q}-\sqrt{6} B(\mathbf{Q} \cdot \mathbf{Q})_{S T}+C \mathbf{Q} \mathbf{Q} \mathbf{Q}+\Gamma_{1} \nabla^{2} \mathbf{Q}\right.} \\
& \left.+\Gamma_{2}(\boldsymbol{\nabla} \nabla \cdot \mathbf{Q})_{S T}\right]
\end{aligned}
$$

is the molecular field conjugate to $\mathrm{Q}$, for a Landau-de Gennes [18] free-energy functional $F$ with up to quartic terms in $\mathbf{Q}$, with phenomenological coefficients $A, B, C$, $\Gamma_{1}, \Gamma_{2}$, and the simplest bilinears in $\boldsymbol{\nabla Q}$. In mean-field theory, the isotropic-nematic transition occurs at $A=$ $A_{*}=2 B^{2} / 9 C$. As in [10-12], we impose plane Couette flow with $\mathbf{u}=y \dot{\gamma} \hat{x}$, ignoring modifications of this flow by order-parameter stresses, and treating $\dot{\gamma}$ as a control parameter, but, unlike in that work, we allow spatial inhomogeneity in the $y$ direction.

As in [12], we rescale time by the relaxation time $\tau / A_{*}$ at the isotropic-nematic transition, $\mathbf{Q}$ as well by its magnitude at that transition, and distances by the diffusion length made from $\Gamma_{1}$ and $\tau / A_{*}$. The ratio $\Gamma_{2} / \Gamma_{1}$ of the Frank constants is, therefore, a free parameter which we have set to 1 in our study. We choose $A=0$ and $\alpha_{1}=0$ throughout, and $\lambda_{k}=-(2 / \sqrt{3}) \alpha_{0}$ and $\dot{\gamma}$ with values as specified in the figures. In the orthonormal basis $[11,12] \quad \mathbf{T}_{0}=\sqrt{3 / 2}(\hat{\mathbf{z}} \hat{\mathbf{z}})_{S T}, \quad \mathbf{T}_{1}=\sqrt{1 / 2}(\hat{\mathbf{x}} \hat{\mathbf{x}}-\hat{\mathbf{y}} \hat{\mathbf{y}}), \quad \mathbf{T}_{2}=$ $\sqrt{2}(\hat{\mathbf{x}} \hat{\mathbf{y}})_{S T}, \mathbf{T}_{3}=\sqrt{2}(\hat{\mathbf{x}} \hat{\mathbf{z}})_{S T}, \mathbf{T}_{4}=\sqrt{2}(\hat{\mathbf{y}} \hat{\mathbf{z}})_{S T}$, we expand $\mathbf{Q}=\sum_{i} a_{i} \mathbf{T}_{i}$ and work with the equations of motion for the five components $a_{i}$ which follow from (1). These equations are numerically integrated using a 4 th order Runge-Kutta scheme with a fixed time step $(\Delta t=0.001)$. For all the results quoted here, a symmetrized form of the finite difference scheme involving nearest neighbors is used to calculate the gradient terms. We have checked that our results are not changed if smaller values of $\Delta t$ are used. We have further checked that the results do not change if the grid spacing is changed and more neighbors to the left and right of a particular site in question are used to calculate the derivative. This gives us confidence that the results quoted here do reflect the behavior of a continuum theory and are not artifacts of the numerical procedure used. We use boundary conditions with the director being normal to the walls. With this, we discard the first $6 \times 10^{6}$ time steps to avoid any possibly transient behavior. We monitor the time evolution of the system for the next $5 \times 10^{6}$ time steps (i.e., $t=5000$ ), recording configurations after every $10^{3}$ steps. We have carried out the study with system sizes ranging from 100 to 5000. In the time-series analysis for the Lyapunov spectrum, we run the simulation till $t=20000$, for a spatial system size of 5000, recording data at spatial points at gaps of 10 .

We focus on the parameter region labeled " $\mathrm{C}$ " or "complex" in $[11,12]$, where the order-parameter dynamics shows temporal chaos. We begin by verifying that the inclusion of spatial variation does not alter the phase diagram radically. To this end, we construct local phase portraits (plotting different components of the order parameter against each other) as a function of the parame- ters entering into the model, the shear-rate $\dot{\gamma}$, and the tumbling parameter $\lambda_{k}$. Shown in Fig. 1 are the local phase portraits for a particular point $x_{0}$ for various shear rates $\dot{\gamma}$, obtained by holding the value of the tumbling parameter fixed at $\lambda_{k}=1.25$. We have checked that the character of the phase portrait (space filling or otherwise) remains intact upon going from one space point to another, though there is no phase coherence between two such portraits. A closed curve corresponding to a limit cycle is seen in the tumbling region of parameter space (denoted by "T") in the figure, while in the $\mathrm{C}$ region of the phase space it is space filling. When one goes away from the $\mathrm{C}$ region of the phase space to the region where the director aligns with the flow, the points reduce to those on a line, and eventually in the aligning regime where the director has already aligned with the flow, it is represented by a point. This assures us that the local dynamics in the spatially extended case is similar to that of the ordinary differential equations of [11,12].

The contribution of the alignment tensor to the deviatoric stress is $[12,19,20] \quad \boldsymbol{\sigma}^{O P} \propto \alpha_{0} \mathbf{G}-\alpha_{1}(\mathbf{Q} \cdot \mathbf{G})_{S T}$ where $\mathbf{G}$, defined in (2), is the nematic molecular field, and the total deviatoric stress is $\boldsymbol{\sigma}^{O P}$ plus the bare viscous stress which is a constant within the passive convection approximation. We therefore look at $\boldsymbol{\sigma}^{O P}$ alone. The spatiotemporal nature of the chaos [21] in this system is seen strikingly, if qualitatively, in the space-time plots of the shear stress (the $x y$ component of $\boldsymbol{\sigma}^{O P}$ ), referred to as $\Sigma$ henceforth, in Fig. 2. Two other figures are instructive, showing the periodic tumbling regime (Fig. 3) and a transition from spatiotemporal chaos to stable flow alignment (Fig. 4). The parameter values at which these are seen, furthermore, correspond well with the phase diagram of $[11,12]$. Our method, therefore, reveals nicely the rich spatiotemporal structure of the phase diagram of this system. In Fig. 4, several distinct events, such as the persistence, movement, and abrupt disappearance of shear bands can be seen, whereas in Fig. 3 the structure is simply periodic. We find that the typical length scale at which banding occurs is a fraction of the system size. In

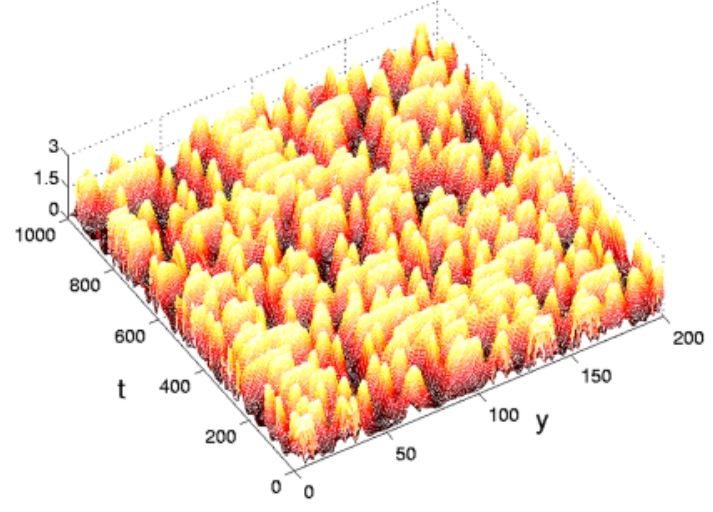

FIG. 2 (color online). Space-time behavior of the shear stress in the chaotic regime, $\dot{\gamma}=3.678$ and $\lambda_{k}=1.25$. Slice taken from a system of size 5000 . 


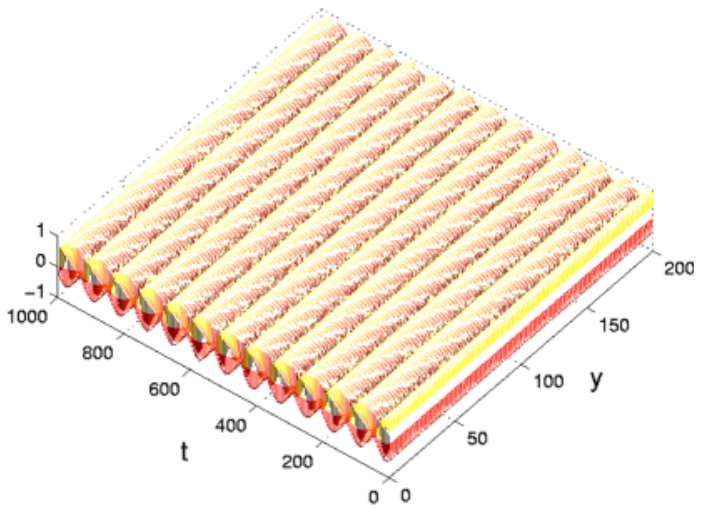

FIG. 3 (color online). Space-time behavior of the shear stress in the tumbling regime, $\dot{\gamma}=5.0$ and $\lambda_{k}=0.9$. Slice taken from a system of size 5000 .

order to characterize better the disorderly structure of the shear bands in Fig. 2, we look at the distribution of band sizes or spatial "stress drops" as follows. At a given time (say, $t_{i}$ ), we define a threshold $\Sigma_{0}=0.8$, a little above the global mean $\langle\Sigma(y, t)\rangle_{y, t}$, and map the spatial configuration to a space-time array of $\pm 1: \tilde{\Sigma}=\operatorname{sgn}\left(\Sigma-\Sigma_{0}\right)$. Figure 5 shows the histogram of the spatial length of intervals corresponding to the + state. We have considered configurations extending over 2500 spatial points, and the statistics is summed over configurations sampled at 5000 times (i.e., $i=1,5000$ ). We see that the distribution of band lengths in the spatiotemporally chaotic regime is fairly broad and roughly exponential in shape, and the time-averaged spatial correlation function of the stress also decays on a length scale of about five bands.

The spatially averaged shear stress shows irregular oscillations in time, but analysis of this signal based on the techniques of [22] does not give reliable results for estimating the correlation dimension $\nu$ mainly because the chaos that we observe is quite high dimensional (embedding dimension [22,23] $m \geq 10$ ). A very long data train is then required for the analysis of the spatially averaged time series to yield a correct value of the corre-

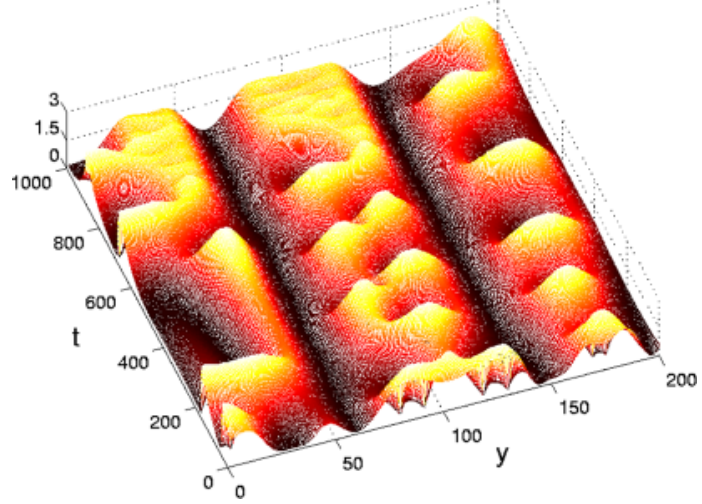

FIG. 4 (color online). Space-time behavior of the shear stress in the chaotic to aligning regime, $\dot{\gamma}=4.1$ and $\lambda_{k}=1.25$. Slice taken from a system of size 5000 .

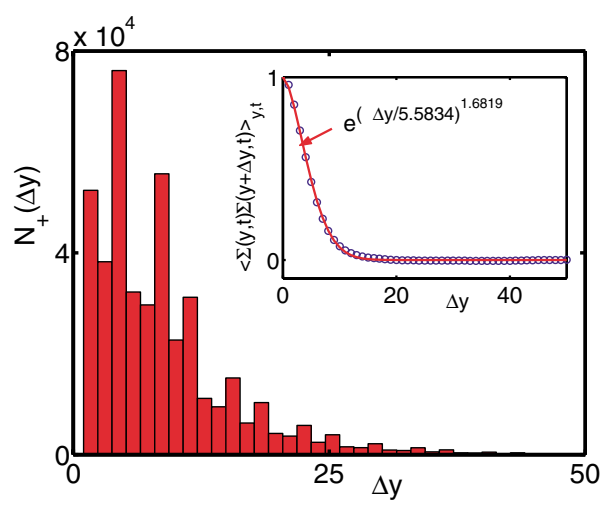

FIG. 5 (color online). Spatial distribution of "stress drops" (corresponding to residence intervals in which the shear stress is above a threshold $\Sigma_{0}=0.8$ ) in the chaotic regime (Fig. 3). Inset shows the spatial autocorrelation function averaged over $t=5000$ (circles) with the best fit to the form $\exp \left[(-\Delta y / \xi)^{\alpha}\right]$.

lation dimensions. We, however, establish conclusively that the oscillatory behavior of the stress and the first normal stress difference shows signatures of spatiotemporal chaos. Instead of trying to implement the correlation-dimension method for our spatially extended problem, we study the Lyapunov spectrum [22,24]. Further, instead of studying systems of ever-increasing size, we look at subsystems of size $N_{s}$ in a given large system of size $N$, i.e., at space points $j$ in an interval $i_{0}<$ $j<i_{0}+N_{s}-1$ where $i_{0}$ is an arbitrary reference point. For spatiotemporal chaos we expect to find that the number of positive Lyapunov exponents grows systematically with $N_{s}$, which is precisely what Fig. 6 shows. For both figures in Fig. 6, we carry out the procedure with two different reference points $i_{0}$ and find essentially the same curves. Furthermore, it has been reported in many studies of spatiotemporally chaotic systems [25] that when calculating the subsystem Lyapunov spectrum for
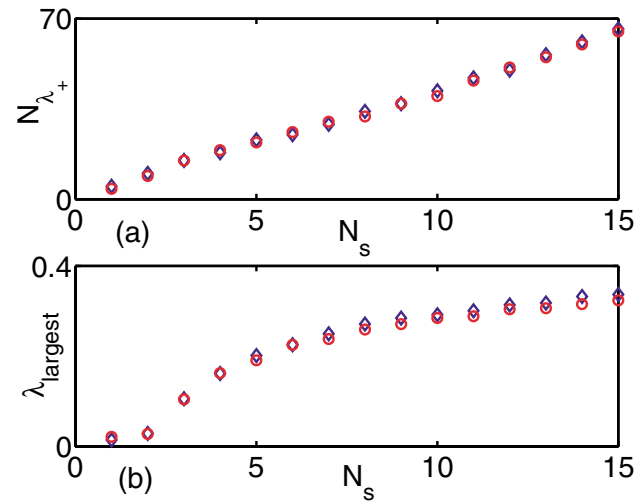

FIG. 6 (color online). Number of positive Lyapunov exponents (a) and the largest Lyapunov exponent (b) as functions of subsystem size $N_{s}$, for $\dot{\gamma}=3.678, \lambda_{k}=1.25$. Embedding dimension for the time series of each space point is 10. Diamonds and circles correspond to data taken from two different regions of space $\left(i_{0}=51\right.$ and $i_{0}=101$, respectively; see text). 
increasing subsystem size $N_{s}$, one finds that the Lyapunov exponents of two consecutive sizes are interleaved, i.e., the $i$ th Lyapunov exponent $\lambda_{i}$ for the subsystem of size $N_{s}$ lies between the $i$ th and $(i+1)$ th Lyapunov exponent of the subsystem of size $N_{s}+1$. A direct consequence of this property is that with increasing subsystem size $N_{s}$, the largest Lyapunov exponent also increases. This is clearly seen in Fig. 6(b).

In summary, we have shown that the stress field in the equations of sheared, nonlinear nematodynamics, in a subrange of the parameter space where flow alignment does not obtain, shows spatiotemporal chaos. Our numerical study of this model, which extends that by [10-12] for the purely temporal behavior, shows irregular, dynamic shear banding, and finds that the number of positive Lyapunov exponents as well as the largest of these grow systematically with system size. Since we do not know what parameter values in our model correspond to the experiments, a detailed comparison to data is not possible at this stage. However, it seems likely to us that the nonlinear relaxation of the order parameter, together with the coupling of nematic order parameter to flow, are the key ingredients for rheological chaos in a variety of problems. Related issues such as the effect of two- and three-dimensional variation, hydrodynamic flow beyond passive advection, the routes to spatiotemporal chaos, and, of course, the role of other degrees of freedom such as variable micelle length and lifetime [9] remain to be explored. Meanwhile, we look forward to quantitative experimental studies of spatiotemporal rheochaos [26].

We thank G. Ananthakrishna and R. Pandit for very useful discussions, and SERC, IISc for computational facilities. M. D. acknowledges support from CSIR, India, and C. D. and S. R. from DST, India, through the Centre for Condensed Matter Theory.

*Present address: Department of Physics, University of Massachusetts, Amherst, MA 01003, USA.

Electronic address: buddho@physics.umass.edu

†Electronic address: moumita@physics.iisc.ernet.in

Electronic address: asood@ physics.iisc.ernet.in

[1] R. Bandyopadhyay, G. Basappa, and A. K. Sood, Phys. Rev. Lett. 84, 2022 (2000).

[2] R. Bandyopadhyay and A. K. Sood, Europhys. Lett. 56, 447 (2001).

[3] R. Bandyopadhyay, G. Basappa, and A. K. Sood, Pramana 53, 223 (1999).

[4] R. Larson, The Structure and Rheology of Complex Fluids (Oxford University Press, New York, 1999).

[5] Intriguingly, J. Dasan, T. R. Ramamohan, A. Singh, and P. R. Nott, Phys. Rev. E 66, 021409 (2002), find rheochaos in sheared hard-sphere Stokesian suspensions, with fluctuations that seem not to decline significantly with increasing system size.
[6] G. Basappa, Suneel, V. Kumaran, P. R. Nott, S. Ramaswamy, V. M. Naik, and D. Rout, Eur. Phys. J. B 12, 269 (1999), report an oscillatory evolution of the storage modulus of defect-ridden lamellar phases under shear treatment.

[7] J.-B. Salmon, A. Colin, and D. Roux, Phys. Rev. E 66, 031505 (2002); J.-B. Salmon, S. Manneville, and A. Colin, cond-mat/0307609.

[8] D. A. Head, A. Ajdari, and M. E. Cates, Phys. Rev. E 64, 061509 (2001).

[9] A. Aradian and M. E. Cates, cond-mat/0310660, and S. M. Fielding and P. D. Olmsted, cond-mat/0310658 [Phys. Rev. Lett. (to be published)]., appeared as e-prints while this Letter was being written.

[10] M. Grosso, R. Keunings, S. Crescitelli, and P. L. Maffettone, Phys. Rev. Lett. 86, 3184 (2001).

[11] G. Rienäcker, M. Kröger, and S. Hess, Phys. Rev. E 66, 040702(R) (2002).

[12] G. Rienäcker, M. Kröger, and S. Hess, Physica (Amsterdam) 315A, 537 (2002).

[13] S. Hess and I. Pardowitz, Z. Naturforsch. 36A, 554 (1981), discuss the hydrodynamics of a spatially inhomogeneous alignment tensor.

[14] N. A. Spenley, M. E. Cates, and T. C. B. McLeish, Phys. Rev. Lett. 71, 939 (1993); J. F. Berret, D. C. Roux, and G. Porte, J. Phys. II (France) 4, 1261 (1994); P. D. Olmsted and C.-Y. D. Lu, Phys. Rev. E 56, R55 (1997).

[15] B. Chakrabarti, Ph.D. thesis, Indian Institute of Science, 2003 (unpublished).

[16] M.W. Johnson, Jr. and D. Segalman, J. Non-Newtonian Fluid Mech. 2, 255 (1977).

[17] More precisely, the deviatoric (traceless symmetrized) stress [4], which is all that matters for effectively incompressible systems.

[18] P.G. de Gennes and J. Prost, The Physics of Liquid Crystals (Clarendon, Oxford, 1995).

[19] D. Forster, Phys. Rev. Lett. 32, 1161 (1974); M. Doi, J. Polym. Sci. Polym. Phys. Ed. 19, 229 (1981).

[20] P. D. Olmsted and P. M. Goldbart, Phys. Rev. A 41, 4578 (1990).

[21] A. Pande, Ph.D. thesis, Indian Institute of Science, 2000, explores spatiotemporal chaos in a wide variety of systems.

[22] R. Hegger, H. Kantz, and T. Schreiber, Chaos 9, 413 (1999); http://lists.mpipks-dresden.mpg.de/ tisean/.

[23] This is estimated using the "false-neighbours" method of M. B. Kennel, R. Brown, and H. D. I. Abarbanel, Phys. Rev. A 45, 3403 (1992).

[24] M. Sano and Y. Sawada, Phys. Rev. Lett. 55, 1082 (1985).

[25] R. Carretero-Gonzalez et al., Chaos 9, 466 (1999); S. Orstavik, R. Carretero-Gonzalez, and J. Stark, Physica (Amsterdam) 147D, 204 (2000); R. CarreteroGonzalez and M. J. Bunner, report (http://wwwrohan.sdsu.edu/ rcarrete/publications/index.html).

[26] P. E. Cladis and W. van Saarloos, in Solitons in Liquid Crystals, edited by L. Lam and J. Prost (Springer, New York, 1992), pp. 136-137, find "director turbulence" in nematics in cylindrical Couette flow. 